This item was submitted to Loughborough's Research Repository by the author.

Items in Figshare are protected by copyright, with all rights reserved, unless otherwise indicated.

\title{
Scenario-based design and evaluation for capability
}

PLEASE CITE THE PUBLISHED VERSION

http://dx.doi.org/10.1109/SYSOSE.2010.5544073

PUBLISHER

(c) IEEE

VERSION

VoR (Version of Record)

LICENCE

CC BY-NC-ND 4.0

REPOSITORY RECORD

Urwin, Esmond N., Colin C. Venters, Duncan J. Russell, Lu Liu, Zongyang Luo, David E. Webster, Michael Henshaw, and Jie Xu. 2019. "Scenario-based Design and Evaluation for Capability". figshare.

https://hdl.handle.net/2134/7238. 
This item was submitted to Loughborough's Institutional Repository (https://dspace.lboro.ac.uk/) by the author and is made available under the following Creative Commons Licence conditions.

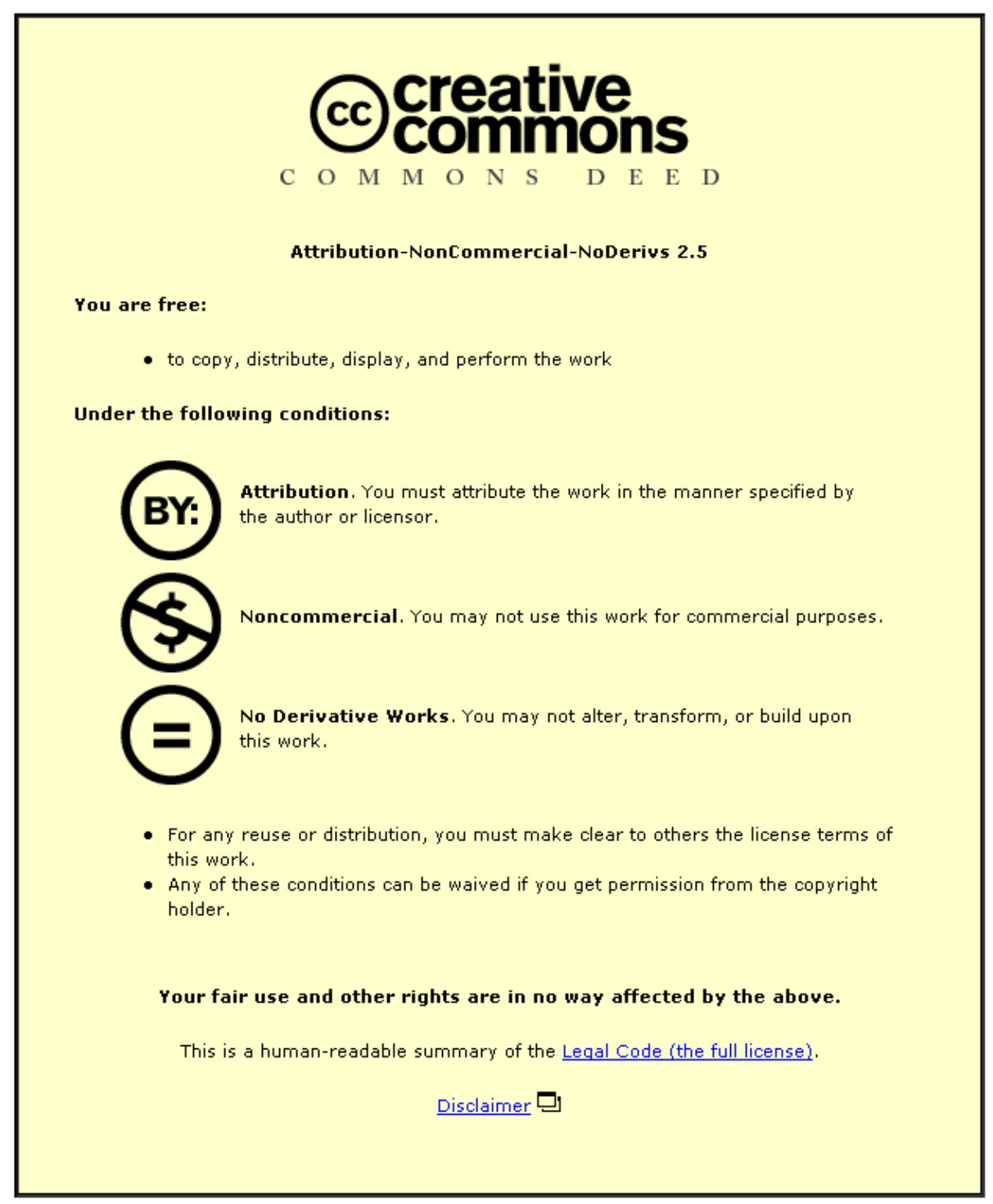

For the full text of this licence, please go to: http://creativecommons.org/licenses/by-nc-nd/2.5/ 


\title{
Scenario-based Design and Evaluation for Capability
}

\author{
Esmond N. Urwin ${ }^{1}$, Colin C. Venters ${ }^{2}$, Duncan J. Russell ${ }^{3}$, Lu Liu ${ }^{4}$, \\ Zongyang $\mathrm{Luo}^{3}$, David E. Webster ${ }^{3}$, Michael Henshaw ${ }^{1}$, and Jie $\mathrm{Xu}^{3}$ \\ ${ }^{1}$ Engineering Systems of Systems Group, Loughborough University, LE11 3TU, UK \\ ${ }^{2}$ Kyungpook National University, Daegu 702-701, South Korea \\ ${ }^{3}$ School of Computing, University of Leeds, LS2 9JT, UK \\ ${ }^{4}$ School of Engineering and Information Sciences, Middlesex University, London, NW4 4BT
}

\begin{abstract}
Abstrac:t Scenarios are frequently used within techniques for planning and designing systems. They are an especially helpful means of visualizing and understanding the incorporation of new systems within systems of systems. If used as the basis for decisions about candidate designs, then it is important that such decisions can be rationalized and quantitative assessment is particularly important. In this paper, an approach for developing complex scenarios, which incorporates the phases of systems development and deployment, is presented and a quantitative method of comparison is described. This approach is based on the development of measures of merit and measures of performance. The techniques are illustrated using cases that are relevant to Network Enabled Capability.
\end{abstract}

Keywords: Scenario based Design, Architectural Evaluation, Capability Assessment.

\section{INTRODUCTION}

$\mathrm{U}$ NDERSTANDING stakeholders' requirements is critical to the success of every system development [1]. Scenariobased design is one of a number of techniques that can be used to elicit requirements [2]. Similarly, scenarios also play a pivotal role in a number of architectural validation methods associated with the process of testing architectures [3]. Scenarios can provide a personalized, fictional story with characters, events, products and environments through which stakeholders can engage in the design process [4]. They also provide system designers with a method to explore ideas and identify the potential ramifications of specific design decisions. The underlying purpose of the scenario approach is to identify likely outcomes to generic circumstances using realistic situations.

Network Enabled Capability (NEC) is the endeavor to enhance [military] capability through the networking of existing and future military assets in order to respond to the rapidly changing conflict environment in which the armed forces must operate [5]. Capability is the ability to achieve a particular military effect in a specific context. NEC requires the integration of independent components, systems, and networks that can evolve and operate in a collaborative and dependable manner. NEC makes demands on the system design that cannot be fulfilled by traditional system engineering design principles [6], because systems no longer operate in isolation. NEC will be realized through networks of systems of systems that are dynamic, large-scale and subject to continual change and evolution.

This paper describes our experience of using scenarios in the context of NEC research. Section II outlines the use of scenario-based design as a method to demonstrate research and engage stakeholders. Section III outlines a scenario-based architectural evaluation framework for the evaluation of candidate architectures and how scenarios can be used to develop a set of measures. Section IV sets out a discussion upon the successes, failures and limitations of the approach, whilst Section V draws together conclusions and outlines future work.

\section{SCENARIO-BASED DESIGN}

Between 2005 and 2009 a multi-institution, multidisciplinary research programme (NECTISE) developed systems engineering approaches to better manage systems for NEC. The programme had a wide scope that included research into high-level architectural approaches, through-life systems management and design decision making, through to specific technological approaches for managing multiple, cooperative UAVs in NEC environments. A major challenge was to create a demonstration (to stakeholders) that integrated the research across these wide-ranging themes.

The stakeholders included government, military, industrial and academic communities working in the maritime, air, and land domains, together with the wider systems engineering community. There were, thus, multiple perspectives to be accommodated within a single demonstration event and a carefully crafted scenario was required in order to show the research benefits to this diverse range of organizations and people.

The scenario-based approach was selected primarily because the storyboard nature would allow stakeholders of varying knowledge in engineering and areas of expertise to appreciate the contextualized application of the research. Carroll [7] states that scenarios can provide sufficient data, information and context to paint a picture that is wholly believable and real enough to be considered viable for experimentation and analysis.

This provides a foundation against which stakeholders can explore the benefits of the research being presented and how it can potentially provide value and future advancement in their specific domains and business. The aim was to therefore create a scenario that satisfied a number of key criteria:

- Include multiple stakeholders' requirements and multiple timeframes. 
- Be representative of NEC and its implications for battle space, the defense industry, UK Ministry of Defence $(\mathrm{MoD})$ and the associated research activities in the programme

- Be sufficiently straight forward to be easily understood by non-experts, but at the same time sufficiently rich to be informative to domain experts.

- Enable the demonstration of multi-disciplinary research outputs.

Following assessment of scenario writing tools and techniques, two were selected as most appropriate for the level of complexity required by the NECTISE programme. The first was The Technical Cooperation Program (TTCP) Guide for Understanding and Implementing Defense Experimentation (GUIDEx) [8], and the second was the Whitworth, et. al. [9] framework for checking the relevance of scenarios to applications under consideration. The starting point for the scenario was to formally assess and consider the main NECTISE project requirements. These set the basic aims and objectives for the scenario to enable its development to fully represent the range of stakeholders' needs. The scenario was developed in an iterative fashion. Requirements were taken from customers (i.e. industry and government) and academics, in terms of research to be demonstrated. Locating research themes within the initial scenario was an important first step. Business experts were invited to assess progress regularly (monthly) and verify the content of the scenario against business requirements. Once the scenario had reached an initial level of maturity, a formal two-day workshop was convened with all of the researchers and academics, along with business experts from BAE Systems, and invited guests from academia, industry, and UK MoD. The outputs of the workshop were the main concepts and storylines for the scenario. Through a series of stakeholder forums and formal design reviews, a stable scenario that incorporated the wide ranging timescales, disciplines, and business objectives was created. Further details of the stakeholder engagement are described in [10] [11].

The basis of the NECTISE scenario is that of a foreign state threatening international airspace with a surface-to-air missile (SAM) weapon system. The operational mission is to neutralize the SAM site to reduce the threat issued against civil air activity. The scenario is composed of four interlinked vignettes, each representing a particular stage of capability development and/or use, and each representing a different timeframe. The main question concerns the development of military capability from inception and planning at the government level, development and assessment within industry, through to use by the military in the operational environment. The capability change in question is an incremental increase in surveillance system capability for monitoring no-fly zones. The four interlinked vignettes are as follows.

- Vignette 4 represents capability planning. At this level, the decisions are taken by the UK MoD with industry having a supporting role. Systems engineering approaches applied are those through which industry can support the UK MoD's capability planning framework. Typically the timeframe for this level is measured in years.
- Vignette 3 is the capability development stage where decisions are made about capability change including the development of options, selection, and change plans. This is applicable to industry and the UK MoD. Typically the timeframe for this is from months to years.

- Vignette 2 looks at the deployment of new, changed, or updated capability. Again, this is applicable to both industry and the UK MoD. The timeframe for this vignette is measured in weeks to months.

- Vignette 1 is concerned with a military operation. Although focused on military needs, this vignette is relevant to industry through identification of systems agility requirements. The timeframe here is hours and minutes.

The purpose for which this scenario was developed was to host research solutions within a viable and realistic context; the purpose is not to show a consolidated solution to the whole scenario. Having outlined how scenario-based design was used to engage a variety of stakeholders in the NECTISE project we now consider the role of scenarios in the identification of measurements for architectural-level validation.

An important output of the two-day stakeholder workshop was a set of NEC-readiness themes, derived through a miniDelphi technique [12] [13] and illuminated through discussion within the multi-disciplinary environment. The NECreadiness themes [14] are essentially cross-cutting themes through the entire research programme and constitute the key parameters that must be managed in order to achieve NECreadiness, i.e. design of systems that are NEC-ready. The themes are: Agility, Interoperability, Dependability, Affordability, Availability, Collaboration, and Knowledge Management (Fig.1). Every research output in the program could be described in terms of these themes, or combinations thereof, and these provided a basis upon which to architect both the scenario and the research outputs within it. Agility is at the centre, because this is the objective of NEC; the interrelated themes must be improved through better systems engineering to enable agility.

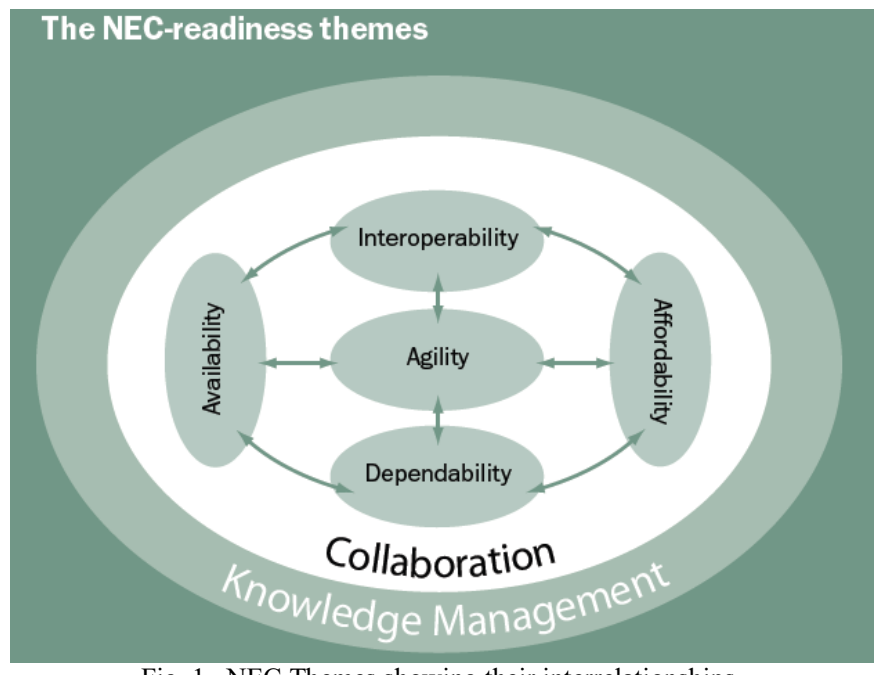

Fig. 1. NEC Themes showing their interrelationships. 
Better understanding of NEC (i.e. how architecture affects capability during the design, development, deployment and evolution of NEC ready systems) may be derived from an evaluation of how different architectures perform. The organisation of the NEC-readiness themes provides a route to formal evaluation of architecture. The themes were represented in MODAF (MoD Architecture Framework). Table I lists the relevant MODAF views for the NEC-readiness themes, but also notes the extent to which the themes may be represented in MODAF

\begin{tabular}{cl}
\multicolumn{1}{c}{ CSF } & \multicolumn{1}{c}{ Summary } \\
Interoperability & Well covered. Many \\
& Views focus on \\
& Interoperability between \\
& Organizations and \\
& Systems.
\end{tabular}

Agility

Several views show how Capability, Systems and Standards will progress over time.

Dependability

Dependability

parameters are not included in MODAF but can be added to existing elements.

Knowledge

Collaboration The MODAF Integrated Dictionary contains the knowledge of the Architecture. Various Views provide the knowledge of the Operational and System and Data Models identify how knowledge of the actual operation will be stored.

MODAF has some views that cover human collaboration and the proposed Human Views provide further support.

Affordability

Not really covered as Cost and Budgets do not exist within MODAF.

Table 1.Mapping of NEC Theme aspects against MODAF framework elements.

\section{Scenario-BASEd ARChitecture EVAluation}

Clements, Kazman, and Klien [15] argue that successful system development and evolution is dependent on making informed decisions at the architectural level. Architecture evaluation is designed to analyze a candidate architecture in order to identify potential risks and to verify that the quality requirements have been addressed in the design [16].
Quality attributes are non-functional requirements of a component or a system. The ISO 9126 quality model provides a structured set of characteristics including functionality, reliability, usability, efficiency, maintainability, and portability against which an architecture can be judged [17]. Each high-level characteristic contains a set of subcharacteristics. For example, reliability contains a set of attributes that are related to the capability of software to maintain its specified level of performance under stated conditions for a stated period of time including maturity, recoverability, and fault tolerance. Each quality subcharacteristic is further divided into entities, which can be verified or measured.

Key quality attributes within NECTISE included agility, dependability, availability, and interoperability. Specific military quality attributes include survivability and lethality. Survivability in this context is concerned with the ability to remain mission capable after a single engagement. Similar to the high level attributes such as dependability, it is a composite attribute that can be measured by susceptibility i.e. the likelihood of being detected, identified, and hit; vulnerability i.e. the effects of being hit by a weapon; recoverability i.e. long term post hit effects, damage control and firefighting, capability restoration or (in extremis) escape and evacuation. Lethality is the effectiveness of a weapon system in all environments against the full spectrum of battlefield threats and measured by the enumeration of survivors after exposure.

A number of scenario-based architectural evaluation methods exist which provide a structured approach to evaluating how well an architecture meets stakeholders' requirements in terms of the quality attributes that the architecture exhibits. The key concept underpinning these methods is a set of scenarios that are important to stakeholders and allow the systems properties to be estimated [18]. However, they differ significantly in their focus and the number of attributes employed. For example, while the Software Architecture Assessment Method (SAAM) [19] and its variants focus on singular quality attributes such as modifiability, flexibility, evolution and reusability other methods such as the Architecture Tradeoff Analysis Method (ATAM) [20], Scenario-Based Architecture Reengineering (SBAR) [21] and the Software Architecture Evaluation Model (SAEM) [22] include multiple attributes which contribute to a better understanding of the strengths and weaknesses of the overall architecture and its constituent parts. The selection of the most appropriate methods is dependent on the context in which the architecture is being evaluated and the quality attributes being addressed [16].

In contrast, Webster, Looker, Russell, Liu, and Xu [23] proposed an architectural evaluation framework (AEF) for NEC that utilizes scenarios in order to understand how to achieve service integration for military capability (see Fig.2). The framework is built on two measures derived from NATO's Measures of Merit (MoM): Measures of Performance (MoP) and Measures of Effectiveness (MoE). MoP verify an individual system against its service specification [6]. The MoP are independent of an operational scenario and allow the results to be compared with systems that provide the same functionality. In contrast, $\mathrm{MoE}$ are dependent on an 
operational scenario. They provide a measurement of how well a system accomplishes its assigned tasks within an operational context and provides a level of confidence in the capability. The formulation of $\mathrm{MoE}$ is dependent on a stakeholder's viewpoint and influenced by the mission or task. Here we can distinguish between the terms measures and metrics. Metrics are a system of measurement through which the merits of an entity can be assessed and measures may contribute to a metric as a set of quantitative values within the system. The separation of $\mathrm{MoE}$ and $\mathrm{MoP}$ allows a decoupling between a conceptual definition of a capability and a physical resource.

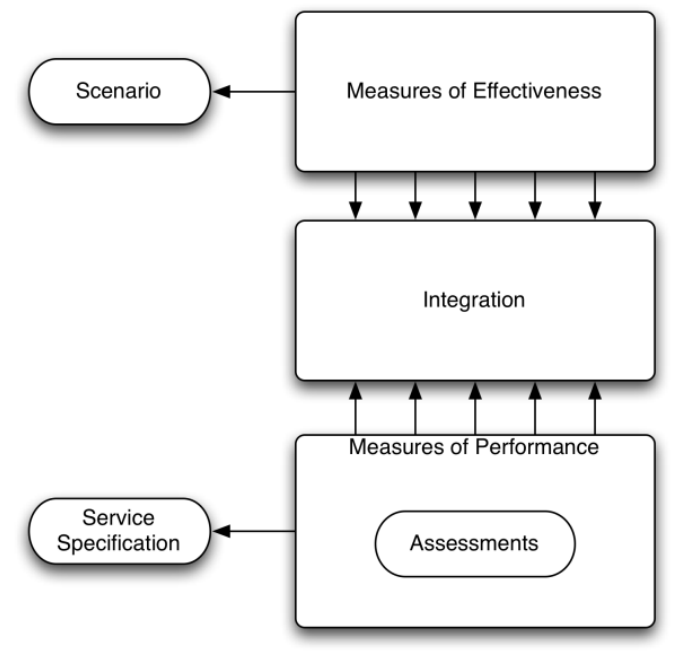

Fig. 2. Architectural Evaluation Framework

Development of the AEF focused on demonstrating how it can be utilized to derive a set of NEC Measures of Effectiveness. A number of scenarios were considered from the perspective of Land and Maritime operational examples: cruise and ballistic missile defence, a time critical target (TCT), and a fire support system (FSS).

In a cruise and ballistic missile defense scenario [24], a small nation island is threatened by large hostile neighbor nation intent on annexing the island. The island's strategic objective is to maintain air superiority over its contiguous waters to the limits of its SAM envelope, to protect Sea Line of Communication (SLOC) and Air Line of Communication until a coalition of friendly forces arrives. Friendly forces, in the shape of two battleships, are positioned to assist the island improve its defense posture against enemy missile attacks. As a result, the battleships also become targets and are prepared to defend against hostiles. In this scenario, there are two competing missions: prevent enemy ballistic missiles from destroying key allied infrastructure targets, and defend against cruise and ballistic missile attacks. The MoE that can be derived from this scenario are the fraction of the critical infrastructure targets that survive the attacks and the fraction of the battleships that survive the attack. In the later case these can be expressed mathematically as the mean number of ships surviving (1) and the cumulative ballistic missile leaks (2).

$$
S_{j}=\frac{1}{2}\left[\left(1-\frac{L_{j}^{(\mathcal{C})}}{N_{L}}\right)+\left(1-\frac{L_{j}^{(\mathbb{D})}}{N_{L}}\right)\right](1)
$$

$$
B_{j=\sum_{k=1}^{j} b_{k}}(2)
$$

In a TCT scenario [24], an attack submarine that was threatening SLOC was killed and a Kilo class submarine is ordered to replace the destroyed submarine. While the time of departure cannot be determined, intelligence confirms the Kilo is preparing for sea. The mission objective is to kill the Kilo on the surface as it emerges from port before it can become a threat. On a separate mission an ISR (Intelligence Surveillance Reconnaissance) submarine has been deployed near the area of interest. The ideal solution is vectoring an F18 to kill the Kilo with a Standoff Land- Attack Missile-Extended Response missile. However, the ISR submarine can be employed to initiate the kill if necessary although this is not considered the optimal solution. The decision on which platform to select is dependent on the decision maker's ability to assess the time available for them to pursue the airborne option expressed as the time on target. Here we can consider the effects of latency in order to make an informed decision (3).

$$
L_{c c}=\frac{1}{1-g(c)} \sum_{i=1}^{\tau} \quad \prod_{j=1}^{d_{i}} \quad\left[\left(1-K_{j}(t)\right)^{\omega_{j}}\right] \frac{\delta_{i}}{\lambda_{i}}(3)
$$

In a fire support system (FSS) scenario [25], a hostile force is threatening a critical node of a Command, Control and Communication (C3) system. The three main elements in the FSS at the battalion level include: the forward observer (FO), the battalion fire direction centre (BFDC), and the field artillery cannon (FAC). The FO receives the initial stimulus by detecting an enemy threat and communicates estimates of the position and velocity of the target, and requests for fire to the BFDC central computer. Each FAC has a receive-only battery display unit (BDU) linked with the $\mathrm{C} 3$ which assists execution of fire plans by receiving and printing firing data for each target that the battery will execute. An enemy tank appears in the field of vision of the FO. The mission is to prevent the tank from attacking the node. The batteries cannot [physically] see the threat and fire according to the directions that are provided by FO data. In this scenario it is assumed that the threat cannot attack the FAC directly and that it will continue to pursue its mission even when fired upon. In addition, the time interval cannot be considered in isolation to yield a MoE but should be considered alongside the doctrine that is feasible at the specific time interval e.g. Look- ShootShoot (LSS) and Look-Shoot-Look-Shoot (LSLS). In this scenario, the MoE is the probability that the node will be defended successfully expressed as the overall probability of kill (OPK) in a given time window where the OPK measures the capability of the FSS to stop the threat and can be computed from the single-shot probability of kill. Our initial work on the development of the AEF suggests that scenarios can be used to derive a set of MoE that would allow reasoning at an architectural-level. However, access to real military scenarios or expertise to develop them is strictly limited due to security restrictions. Nevertheless, it demonstrates that military personnel could use the AEF to identify measurements from scenarios for architectural-level validation. 


\section{DISCUSSION}

With the nature of military operations becoming ever more collaborative, there is a requirement for more interoperable and NEC capable systems that allow those forces to work together more effectively to better achieve planned missions and goals within theatre. But what is the best way to understand, measure, and assess how these NEC ready systems will perform, and what long-term acquisition and planning decisions should be made to accommodate such systems? One way is to apply a scenario-based design process to create viable situations and contexts. This allows the issues to be explored and assessed; from this an understanding can be gained about the appropriate choices to be made for a given scenario, the systems involved and the possible outcomes. The way in which the scenario based design technique becomes relevant for the assessment of NEC has been through the application of the NEC themes. When creating or building a scenario, each aspect of the themes can be represented within it. This means that it can be assessed and measured against, for example, interoperability, agility or dependability. These themes in turn link directly to MODAF meaning that scenarios can thus be used to assess architectural frameworks for elements of risk, measures of merit and performance.

The work accomplished within the NECTISE research programme has brought about the mapping of the MODAF viewpoints to the relative NEC themes (see table 1). Such a relationship map allows scenarios and vignettes to be assessed against the criteria within each respective MODAF viewpoint, thereby bringing about the ability to evaluate capability for legacy, current and proposed systems against a context. Add to this the ability to apply the AEF and it provides a new way in which capability and architecture can be evaluated so as to meet stakeholder needs. This is illustrated in fig.3 which shows the interaction between the NECTISE scenario vignettes, the NEC themes and MODAF.

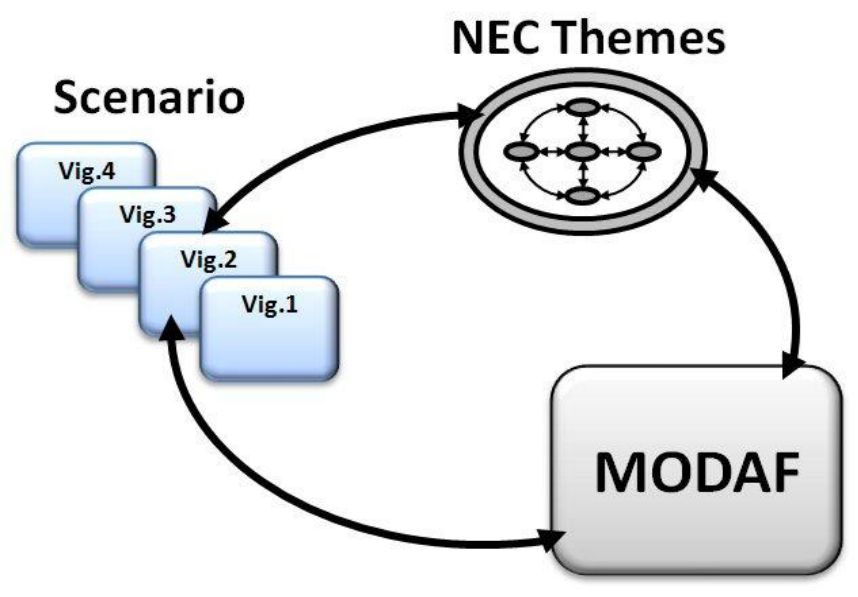

Fig. 3. Using scenarios for capability assessment.

It must be noted that whilst this paper has focused on a NEC specific approach to scenario development and assessment, it is perfectly feasible to apply such an approach and method to non-NEC aspects for understanding stakeholder requirements and capability evaluation.

\section{SUMMARY AND CONCLUSIONS}

Scenarios provide an important means through which proposed systems may be assessed prior to development or deployment, but decisions about the best architectures to provide solutions requires quantitative evaluation. The approach described herein provides such a quantitative assessment method. In summary, a stakeholder engagement plan is developed through which a composite scenario is developed. The scenario is composed of vignettes that contextualize the principal phases of systems development to meet a capability need. One or more 'operational' vignettes must be included to test the deployed system. The system attributes are described through an appropriate set of 'ilities that provide linkage from one vignette to the next. An assessment framework based on measures of Merit and Measures of Performance has been described, through which candidate architectures can be quantitatively compared, thus providing a rational basis upon which to make technical decisions about solution systems to support capabilities.

\section{ACKNOWLEDGMENT}

The work reported in this paper has been supported by the NECTISE program, which was jointly funded by BAE Systems and the UK Engineering and Physical Sciences Research Council Grant EP/D505461/1.

\section{REFERENCES}

[1] I. Sommerville, Software Engineering: Addison-Wesley, 2006.

[2] G. Kotonya and I. Sommerville, Requirements Engineering: Process \& Techniques: John Wiley \& Sons, 1998.

[3] H. J. Reekie and R. J. McAdam, A Software Architecture Primer: Angophora Press, 2003.

[4] J. Preece, Y.Rogers, H. Sharp, D. Benyon, S. Holland, and T. Carey, Human-Computer Interaction: Addison-Wesley, 1994.

[5] UK Ministry of Defence, "Network Enabled Capability," Ministry of Defence UK 2005.

[6] D. Russell, N. Looker, L. Liu, and J. Xu, "ServiceOriented Integration of Systems for Military Capability," in The 11th IEEE International Symposium on Object/component/service-oriented Real-time Distributed Computing (ISORC08), Orlando, USA, p. 33, 2008.

[7] J. M. Carroll, "Five Reasons for Scenario Based Design," Interacting with Computers, vol. 13, pp. 43-60, 2000. 
[8] Guide for Understanding and Implementing Defense Experimentation (GUIDEx): The Technical Cooperation Program, 2006.

[9] I. R. Whitworth, S. J. Smith, G. N. Hone, I. McLeod, "How do we know that a scenario is 'appropriate' ", 11th International Command and Control Technology Symposium, Cambridge, UK, 26-28th September, 2006.

[10] Urwin, E.N., Henshaw, M.J.C., "Managing Innovation A Scenario Based Planning Approach", The 5th International Conference on Advances in Production Management (APMS2009), Bordeaux, France, 19-23 September, 2009.

[11] Henshaw, M.J.C., Gunton, D.J., Urwin, E.N., "Collaborative, academic-industry research approach for advancing systems engineering", The 7th Annual Conference on Systems Engineering Research (CSER2009), Loughborough, UK, 20-22 April, 2009.

[12] Cuhls, K., "Delphi Method", Fraunhofer Institute for Systems and Innovation Research,http://www.unido.org/fileadmin/import/16959_Delp hiMethod.pdf

[13] Dalkey, N. \& Helmer, O. 1963, "An experimental application of the Delphi method to the use of experts", Management Sci., 9(3), 458-467.

[14] Neaga, E.I. and Henshaw, M.J.d., "NEC Themes: A Conceptual Analysis and Applied Principles", Realising NEC Conference, Leeds, UK, October 2008.

[15] P. Clements, R. Kazman, and M. Klien, Evaluating Software Architectures: Methods and Case Studies: AddisonWesley, 2002.

[16] L. Dobrica and E. Niemelae, "A Survey on Software Architecture Analysis Methods," IEEE Trans. on Software Engineering, vol. 28 (7), pp. 638-653, 2002.

[17] "ISO/IEC 9126-1:2001: Software Engineering: Product Quality: Part 1: Quality Model," International Organization for Standards, 2001.

[18] N. Rozanski and E. Woods, Software Systems Architecture: Working With Stakeholders Using Viewpoints and Perspectives: Addison Wesley, 2005.

[19] R. Kazman, G. Abowd, L. Bass, and P. Clements, "Scenario-Based Analysis of Software Architecture," IEEE Software, Nov, pp. 47-55, 1996.

[20] R. Kazman, M. Klein, M. Barbacci, H. Lipson, T. Longstaff, and S. J. Carrieare, "The Architecture Tradeoff Analysis Method," in ICECCS '98: Proceedings of the Fourth International Conference on Engineering of Complex Computer Systems Monterey, CA, USA: IEEE Computer Society, 1998, pp. 68-78.
[21] P. O. Bengtsson and J. Bosch, "Scenario-Based Architecture Reengineering," in ICSR: Proceedings of the Fifth International Conference on Software Reuse, Victoria, BC, Canada, 1998.

[22] J. C. Duenas, W. L. de Oliveira, and J. A. de la Puente, "A Software Architecture Evaluation Model," in Proceedings of the Second International

ESPRIT ARES Workshop. Lecture Notes in Computer Science 1429 Las Palmas de Gran Canaria, Spain: Springer, 1998.

[23] D. Webster, N. Looker, D. Russell, L. Liu, and J. Xu, "An Ontology for Evaluation of Network Enabled Capability Architectures," in RNEC'08: Realising Network Enabled Capability, Leeds, UK, 2008.

[24] W. Perry, R. W. Button, J. Bracken, T. Sullivan, and J. Mitchell, "Measures of Effectiveness for the Information-Age Navy," RAND, 2002.

[25] P. H. Cothier and A. H. Levis, "Timeliness and Measures of Effectiveness in Command and Control," IEEE Trans. on Systems, Man and Cybernetics, vol. SMC-16, pp. 844-853, 1986. 